\title{
A numerical code for underwater noise propagation
}

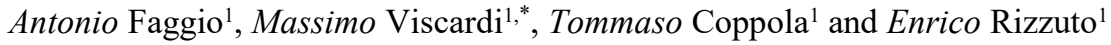 \\ ${ }^{1}$ Department of Industrial Engineering - University of Naples "Federico II" - Via Claudio, 21 - 80125 Naples _Italy
}

\begin{abstract}
A growing attention has been recently devoted to the impact of the underwater noise field generated by shipping activities on the marine fauna. While in international treaties and conventions this problem is now well treated, from a technical point of view the quantification of the impact of underwater noise emissions from the ship is not yet consolidated.

The program created allows the user to identify the sound rays generated by source and, between these, to find the beam nearest a receiver calculating the Trasmission Loss (TL). The user enters as input data the source position, the Sound Pressure Level (SPL) of the source, the depth of the backdrop, sound velocity profile, thickness of the layers in which the water column is divided, salinity, temperature and $\mathrm{pH}$ of sea water and the frequency of emission of the source to characterize the propagation environment. Through the Ray Tracing Theory, a technique of optical geometry that is based on the calculation of the path made by light the rays are shown on the screen. Then the rays closest to the receiver are identified and the program calculates the sound loss, divided into Geometrical Loss (due to distance between the source and the receiver), Dissipation Loss (due to the characteristics of the propagation environment) and Reflection Loss (due to the surfaces that delimit the field).In output the program will show the propagation field of the Sound Pressure Level through which in the future it will be possible to identify the emission limits to protect the marine fauna.
\end{abstract}

\section{Introduction}

The underwater noise generated by the many coastal and offshore anthropic activities is now universally recognized as a form of cross-border marine pollution (UNCLOS 3, United Nations Convention on the Law of the Sea): it consists of continuous sounds (such as perforations, traffic naval) and impulsive (like battipali, geoseismic investigations) that are added to the noise normally present in the marine environment. It must be specified that the characteristics of the noise caused by the ships vary depending on the type of boat, its size, the type of propulsion, its design and cruising speed: it has been proved, however, that the only sound produced by the cavitation of the The propeller can spread to a radius of hundreds of kilometers around the ship that generated it. On the other hand, as regards the research activity of fossil fuel deposits, the increasingly frequent use by the industry of the seismic prospecting system, which is ecologically destructive, must be considered.

In 2008 the European Community, through the "Marine Strategy Framework Directive", considered noise as a qualitative parameter of the marine environment, requiring Member States to tackle the problem by acting as a precautionary measure and avoiding any kind of cross-border pollution.

In the absence of the possibility to regulate the noise resulting from navigation through the adoption of a new ad hoc annex of the MAR.POL. convention, since the same applies only to forms of pollution from substances and not from forms of energy (art. 1), it seems that the most appropriate venue for the elaboration of an international regulation on the matter remains the IMO (International Maritime Organization). In fact, in September 2010, the IMO invited the ISO (International Standard Organization) to develop an international standard aimed at measuring underwater noise produced by ships, considered as the first form of submarine noise pollution (ISO, 2011) .

\section{Underwater acoustic}

The sound is a variation of pressure that propagates in an aeriform, liquid or solid elastic physical medium. The propagation of an acoustic wave is a mechanical energy transport mechanism: this, in fact, presents itself as a succession of compressions and rarefactions of the medium; the acoustic signal is therefore associated with pressure variations.

\subsection{Speed of sound}

The sound propagates in water at a higher speed than air propagation (about $1500 \mathrm{~m} / \mathrm{s}$ in water compared to about $340 \mathrm{~m} / \mathrm{s}$ in air), which makes sea water a favorable medium for the transmission of sound .The speed of sound can be obtained from static measurements on the fluid. In fact, for some years, this method gives the values of the speed of sound in water more accurate on a wide range of pressure, temperature

\footnotetext{
* Corresponding author: massimo.viscardi@unina.it
} 
and salinity values. This is based on the following report, attributed to Newton:

$$
c=\sqrt{\frac{K}{\rho}}
$$

where "c" is the speed of sound, " $\rho$ " is the density of the medium, " $\mathrm{K}$ " is the compression module, a parameter that describes, at a macroscopic level, the binding force between the molecules of the medium, in Pascal.

In general it can be said that the speed of sound in relation to depth is influenced by three independent variables:

1) Temperature; 2) Salinity (weight of solids dissolved in grams contained in $1 \mathrm{~kg}$ of sea water, expressed in parts per thousand); 3) Pressure.

Many authors have published formulas for the evaluation of the speed of sound according to these parameters: to date, the most useful from the practical point of view, which applies in conditions of common scenario, such as water temperature between 0 and $32^{\circ}$ C , salinity level between 22 and 45 ppt (Parts Per Thousand) and depths below $1000 \mathrm{~m}$, is the simplification of the model of Del Grosso implemented by Medwin (Urick, 1979):

$c=1449.2+4.6 T-0.055 T 2+0.00029 T 3$

$+(1.34-0.010 T)(S-35)+0.016 D[\mathrm{~m} / \mathrm{s}]$

where "D" is the depth in meters, considered increasing with increasing depth, " $\mathrm{T}$ " is the temperature in degrees Centigrade, "S" is the salinity in parts per thousand.

It has been shown that the speed of sound increases by $4.5 \mathrm{~m} / \mathrm{s}$ for each unit increase in temperature and, for this reason, the speed profile for the "hot" months changes significantly compared to that for the "colder" months. The absorption of solar energy decreases rapidly with depth, but the variation of the water temperature does not follow the absorption curve due to the wind which, generating the wave motion, causes a mixing of the surface layer (mixed layer). Immediately thereafter is the thermocline level, which is the transition state between the mixed surface layer and the isothermal deep water layer. Below the thermocline level, as seen in Figure 1, the temperature remains constantly low. (Tamà, et al., 2015)

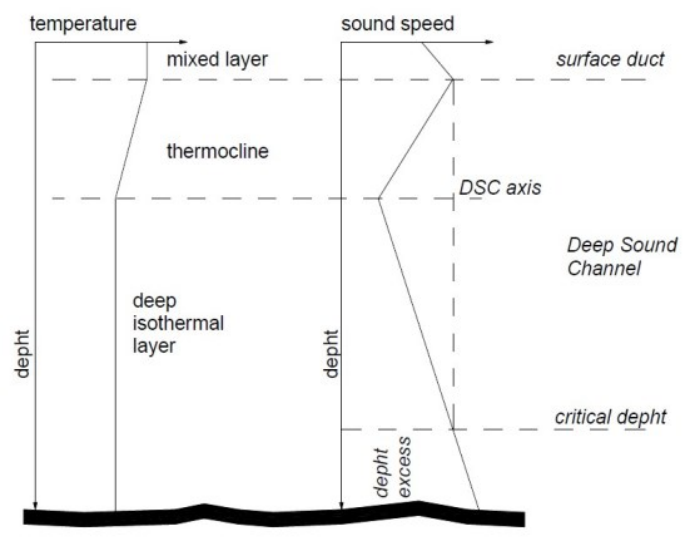

Fig. 1. Speed of sound as function of sea depth

\subsection{Intensity Loss}

When an acoustic wave propagates, the most evident effect is the loss of intensity caused by geometric loss (diffusion) and absorption of acoustic energy. This loss of propagation (also referred to as TL, transmission loss) is a fundamental parameter in an acoustic system.

There are further losses due to the fact that water is not a homogeneous medium, but, on the contrary, characterized by the presence of bubbles, plankton and particulate. These are all processes where the energy radiated by a sound source is redistributed into space, except for the absorption, which represents the conversion of elastic energy of the sound wave into heat and results in a heating of the medium in which it occurs. In an absorbing medium, a fraction of sound intensity is surely lost and turned into heat in a certain distance. Indicated with " $\mathrm{x}$ " the distance you can write:

$$
10 * \log \left(I_{2} / I_{1}\right)=-\alpha\left(x_{2}-x_{1}\right)
$$

This law is also known as Beer's law and, generally, for submarine sound " $\alpha$ " is expressed in decibels per kilometer $(\mathrm{dB} \cdot \mathrm{km})$ to avoid values that are too low. The known values of the attenuation coefficient are referred to a range from $20 \mathrm{~Hz}$ to $60 \mathrm{kHz}$ in the open sea, there are no data related to this field.

The coefficient " $\alpha$ " depends on the frequency: this dependence, however, is made complicated by the fact that, for each frequency band, different processes are involved. Below is shown in Figure 2 of the curves that approximate the variation of " $\alpha$ " with the frequency in sea water in a range from $1 \mathrm{~Hz}$ to $10 \mathrm{MHz}$.

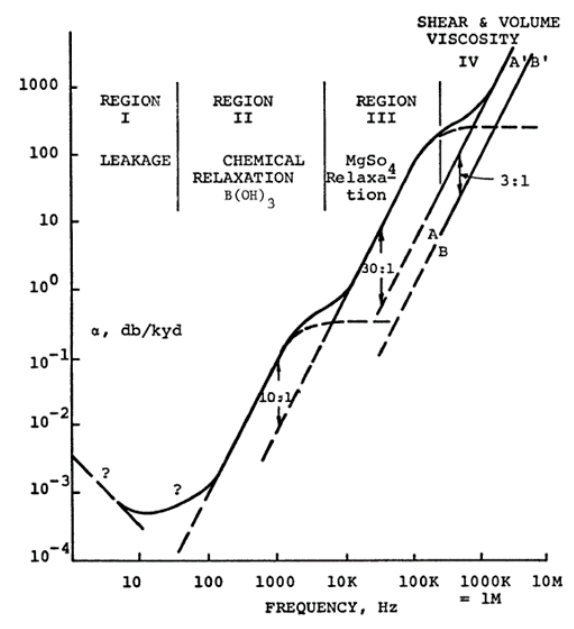

Fig. 2. Beer's law

As can be seen, the diagram shown in Figure 2 is divided into regions, each of which is identified by the main process involved in the dispersion of sound. In Region 4 (at very high frequencies), viscosity and heat conduction are predominant: the first has been demonstrated by Rayleigh (Urick, 1979), while for the second a loss is created due to a heat flow that compresses the sound wave. In this area, however, the coefficient " $\alpha$ " is a function of the frequency square, represented by the A- 
A1 curve: for sea water, however, consider also the presence of dissolved ions in water, whose contribution is predominant in Region 3 but is also clearly seen in Region 4.

Referring to Beer's law, the length of the acoustic wave's attenuation in water is defined as the distance " $\mathrm{X}$ " from the position of the source such that the intensity of the signal is reduced by a factor " 1 / e". Because of the small attenuation value referred to by frequency region of the order of $\mathrm{kHz}$ (about $1.5 \cdot 10^{-8} \cdot \mathrm{f} 2$ ), it is found that, for such frequencies, " $\mathrm{X}$ " is of the order of kilometer; for frequencies of the order of $10^{2} \mathrm{kHz}$, instead, " $\mathrm{X}$ " is of the order of $10^{4} \mathrm{~km}$. With reference to similar considerations, we speak of water transparency with respect to acoustic emission or, if one prefers, one can say that the sea is opaque to transmission.

\subsection{Refraction}

The Snell law, which describes the mode of refraction and reflection of a ray in the transition between two different refractive index means, allows to represent the trend of a sound ray between two homogeneous fluids with different sound velocities.

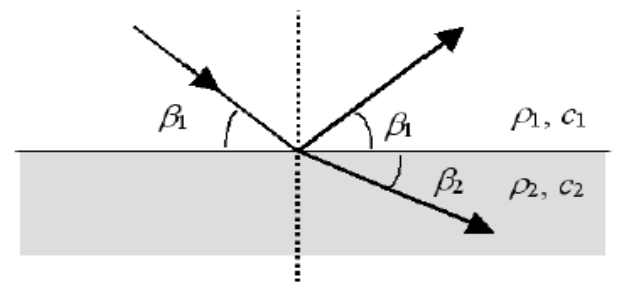

Fig. 3. Snell law

$$
\frac{\cos \beta_{1}}{c_{1}}=\frac{\cos \beta_{2}}{c_{2}}
$$

The limit angle is given by the equation:

$$
\beta c=\arccos \left(c_{1} / c_{2}\right)
$$

known as the critical angle for interference. For angles smaller than the critical angle there is only reflection, making refraction impossible in the second medium. Snell's law can also apply to a series of levels with constant speed: continuous changes in the speed of sound can change the initial direction of the wave.
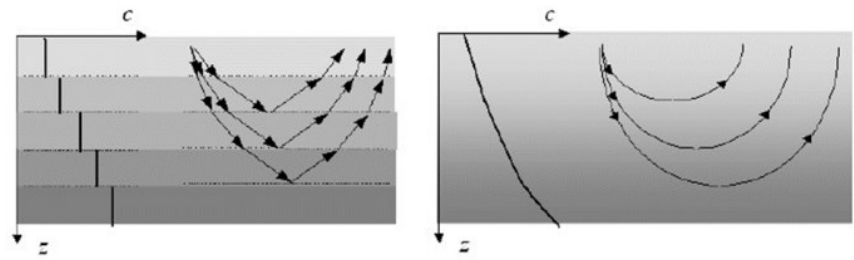

Fig. 4. Acoustic ray deviation by diffraction effect

\subsection{Reflection and dispersion from free surface}

The wave surface affects the propagation of sound at sea in different ways. When a sound wave hits the still surface of the sea, a part of energy is reflected back into the water, and a small part, about $0.02 \%$, crosses the surface and is dispersed. When the sea, instead, is moved, the propagation of the incident waves follows different paths: the relationship between the waves returning from the surface and the incident waves is less than unity and the sound intensity of the transmitted sound is reduced. When the sea is very rough, the reflected sound in the direction of specular propagation is very small.

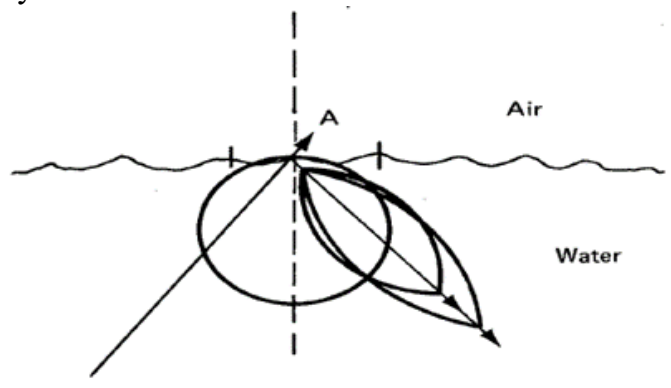

Fig. 5. Reflection by the free boundary

A measure of the "roughness" of the wave surface is the Rayleigh "R" parameter defined as:

$R=2 k h \sin \varphi$

where " $k$ " is the sound wave number, set equal to $2 \pi / \lambda$, " $\mathrm{h}$ " is the height of the wave and " $\varphi$ " is the grazing angle. If $\mathrm{R}<<1$, then the surface is considered quiet, if $\mathrm{R}>>1$ then the sea is rough.

\subsection{Reflection and dispersion from the seabed}

The seabed has many effects on the submarine sound propagation: it reflects and disperses the sound, it produces a model of interference in the radiation from the source. The reflection of the seabed is more complex than that of the wave surface for several reasons:

1. The backdrop can have very different acoustic properties as it can vary from hard rock to soft mud;

2. It is often stratified, with a density and a speed of sound that change gradually or suddenly with depth;

3. It is very inhomogeneous, with large variations of characteristics in small distances;

4. The sound can penetrate into a sedimentary bottom and be reflected from a lower level or from a steep gradient gradient in the sediment.

The sound intensity coefficient reflected by a seabed is:

$$
\mu=\mu_{0} \exp \left(\frac{-4 \pi h \sin \vartheta}{\lambda}\right)^{2}=\mu_{0} e^{-R^{2}}
$$

where $" \mu_{0} "$ is the reflection coefficient with smooth background and depends on density, rigidity, absorption, 
compressibility and layering of the bottom; the exponential represents the roughness of the backdrop, with Rayleigh's "R" parameter that, to date, is almost unknown. For this reason the loss of sound intensity is very complex to evaluate.

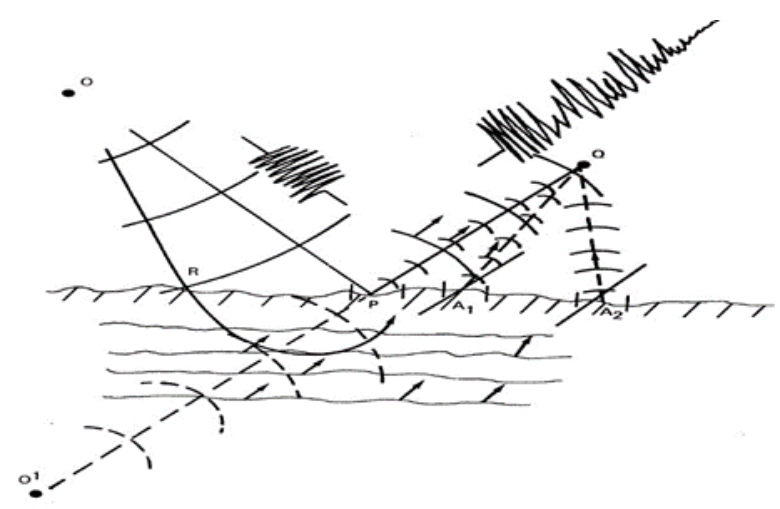

Fig. 6. Complex noise paths close to the seabed

\subsection{Direct paths}

Sound waves that travel directly from the sound source to the receiver without interactions with the wave surface or the seabed are called direct sound waves. The direct path is always the first propagation path available and includes the initial part of all propagation paths, but is generally only available for a small distance.

The Shallow Water, in the acoustic sense, exists everywhere the propagation is characterized by numerous reflections both with the wave surface and with the seabed. Generally, an area with a depth not greater than 200 meters can be considered as shallow water.

The most important feature of sound propagation in this area is represented by the velocity profile trend, which has a negative or approximately constant gradient as the depth changes. This means that the losses over long distances are mainly due to the interference of the sound waves with the surface and the bottom, which involves great loss of energy caused by the coefficients of attenuation of the surfaces.

\section{Predictive software for underwater noise propagation}

\subsection{Code overall specifics}

The present work includes a development part of a calculation program dedicated to modeling the phase of sound propagation in water, from the source, considered as point-like and placed near the free surface, to a generic receiving position in the fluid mass.

The program allows the operator to represent the sound beams emitted by a source and, among these, identify the one closest to a certain receiver by calculating the sound loss (TL).

As input data that the program receives from the user there are:

- Position of the source;

- Sound pressure value of the source;

- Depth of the backdrop;

- Thickness of the layers in which the water column is divided;

- Speed of sound on the free surface;

- Sound speed gradient when the depth changes;

- Minimum angle of beam emission, maximum angle and interval between these;

- Salinity of sea water;

- Water temperature;

- Frequency of emission of the source;

- $\mathrm{pH}$ of the sea.

These input data, which obviously vary from sea to sea, allow to define the water column to be considered for the propagation of sound rays and to define the water absorption coefficient, considered a non-homogeneous liquid.

At the second step the program elaborates the propagation paths of the sound beams using the Ray Tracing technique.

At the third point the receivers are defined, which can be for example hydrophones, going to divide the depth of the backdrop regularly. In order to identify the radius closest to a certain receiver, a tolerance can be defined (at the discretion of the user) that may vary depending on the distance from the source (it is clear that the closer we are to the source and the greater the beam thickening) .

Once the radius closest to a certain receiver has been identified, the fourth point is passed, that is the calculation of the Transmission Loss, that is to say, what is the loss, in terms of energy, up to the receiver in question.

To take into account the absorption and transmission characteristics of the seabed, an additional term has been added to the TL formula described in chapter 1 and shown below:

$T L=20 \log (R)+\alpha R[d B]$

The additional rate was estimated at 0.5 , so a contribution of $3 \mathrm{~dB}$ was added to the overall $\mathrm{TL}$ formula. For the wave surface, however, only the reflection was considered since, as previously said, the transmitted sound rate is equal to $0.02 \%$ (due to the great difference in density between air and water): in this case the additional term it has been neglected.

The program ends, at point five, with the calculation of the Sound Pressure Level, referring to the direct field, field generated by the various reflections and adding them in energetic terms so as to obtain the overall sound field due to a certain point source. 


\subsection{Ray Tracing and Code implementation}

It is a general technique of optical geometry but is also used for the determination of sound beams.

Starting from a point sound source in a medium with constant sound velocity, the sound beams have a rectilinear course as they move away from the source. If the speed of sound is not constant, the sound beams are no longer rectilinear but tend to bend.

The multipath were developed considering the linear propagation speed with the depth:
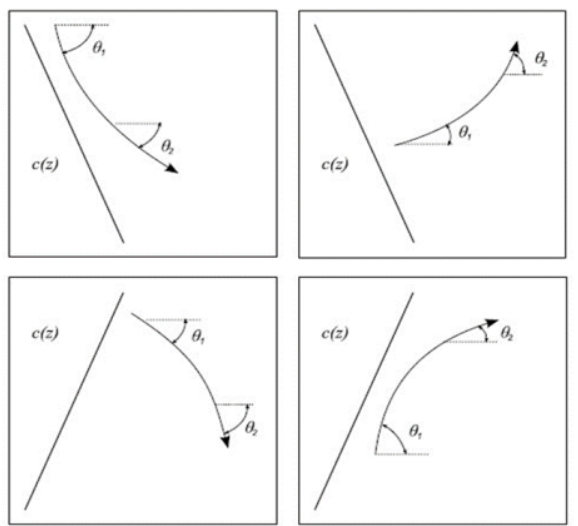

Fig. 7. Different paths curvature

As can be seen from the figure, the trend of the radius varies with the variation of the initial angle (positive if below the horizontal, negative above) and of the velocity trend (increasing or decreasing with the depth).

For each level in which the water column has been divided, according to Snell's law, the incident and transmission angles of the sound beam have been considered, verifying that these were always greater than the critical radius: otherwise, in fact, the ray is totally reflected, reversing the propagation direction.

Along the code, starting from the source, the radius, with a certain angle chosen by the user, crosses the first layer of the water column and, as a result of the speed variation, changes the propagation angle.

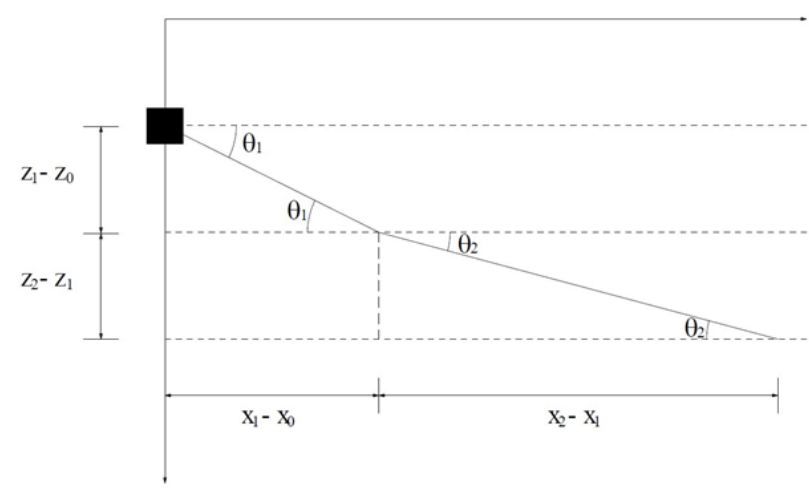

Fig. 8. Acoustic ray curvature

In the angle of refraction is lower than the critical one, so that the minimum conditions for reflection no longer exists, the ray travel as follow:

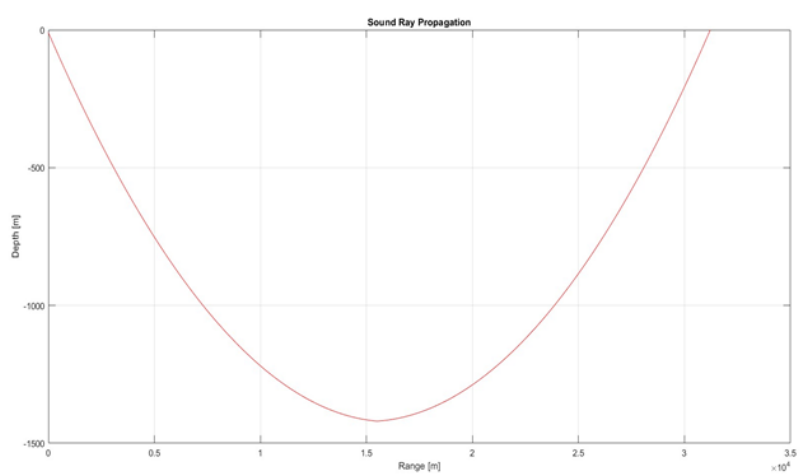

Fig. 9. Acoustic ray curvature below critical angle

The next step was to identify the reflections on the seabed and on the free surface: the angle of reflection, in this case, is equal to the angle of incidence, specularly to the normal at the bottom or free surface at the intersection point.

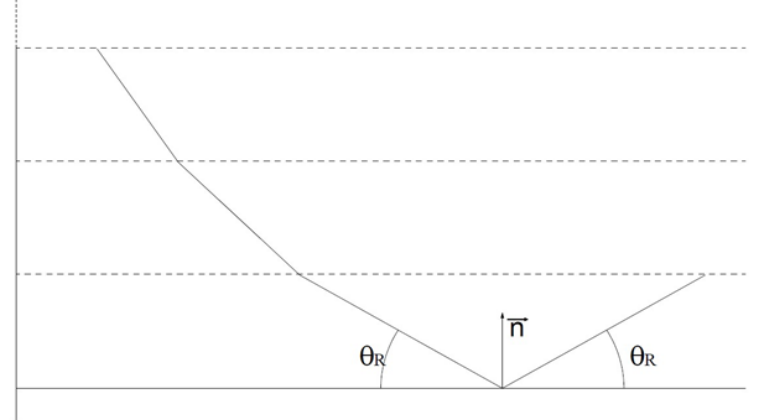

Fig. 10. Acoustic ray curvature below critical angle

This procedure has been iterated up to consider four reflections (including those due to a critical angle), obtaining a situation like this:

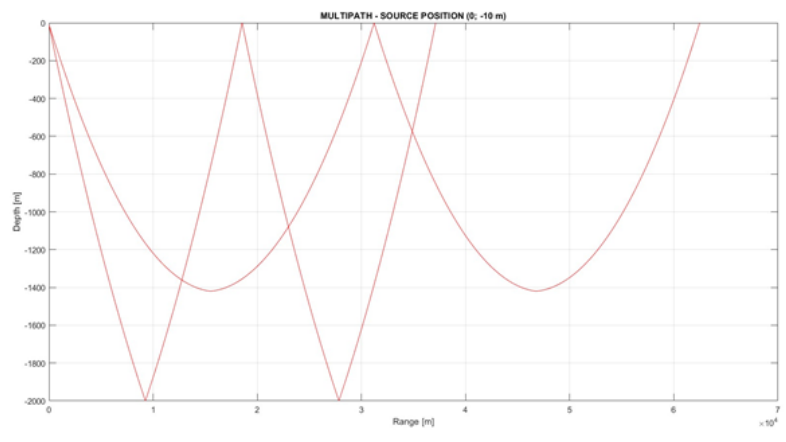

Fig. 11. Acoustic ray propagation

\subsection{TL evaluation}

Once the part of the program relating to the propagation of sound beams has been completed, for each receiver placed in any zone, the radius closest to it has been evaluated and, consequently, the TL corresponding to the single receiver has been calculated. 
By calculating the path length to the point of the beam closest to the receiver, the "TL" was evaluated as spherical, then:

$T L=20 * \log R[d B]$

with "R" beam length up to the receiver.

To add to this "TL" there is the contribution due to the absorption coefficient, due to the non-homogeneity of the water, variable with the backdrop, frequency, temperature, salinity and $\mathrm{pH}$.

$$
T L=20 * \log R+\alpha R[d B]
$$

As already specified, for the incident rays on the bottom it has been considered a coefficient of absorption and transmission equal to 0.5 , ie it involves an addition of 3 $\mathrm{dB}$ to the calculation of the TL.

$T L=20 * \log R+\alpha R+n 3[d B]$

with "n" number of reflections on the bottom.

To find the radius closest to the receiver, the distance of each beam from the receivers was evaluated, taken one at a time. If this distance is less than a certain tolerance, variable when the range taken into consideration varies (with variability set by the user) because it is understood that it can be more precise short rather than long distances, then the length of the radius is calculated identified up to the receiver in analysis.

The receivers have been organized in a regular grid so that the propagation of the SPL can be identified from the source.

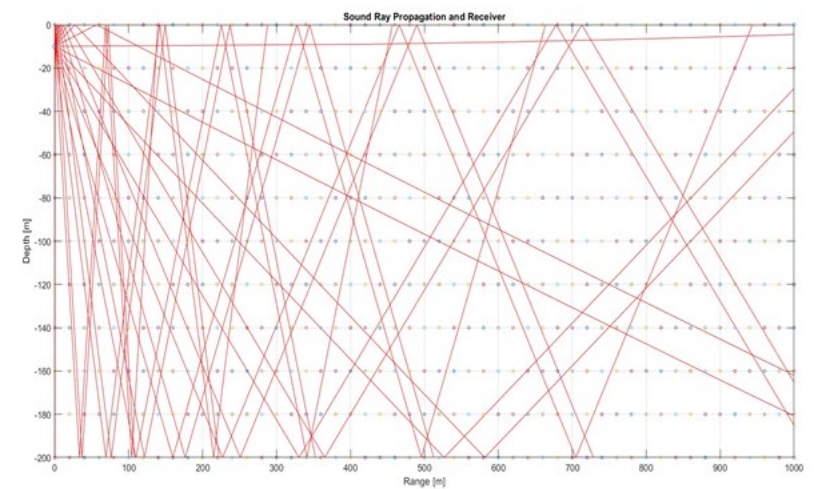

Fig. 12. Receivers matrix

\section{Main Simulation Results}

Some simulations have been performed, having located a noise sources of $100 \mathrm{~dB}$ at $15 \mathrm{mt}$ from the free sea surface.

Below are the results obtained by adding first direct field and field generated by the reflected rays from the free surface, then direct field and field generated by the reflected rays on the bottom.
The direct field iso-pressure curves follow spherical profiles, as expected from the attenuation characteristics by divergence.

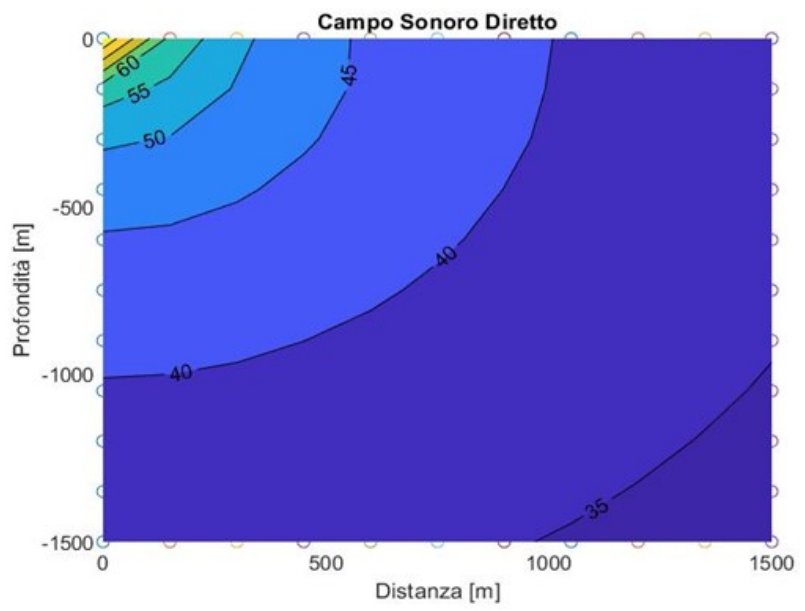

Fig. 13. Direct field propagation

Each point of contact with the hair turns into a virtual emission source, which falls within the propagation domain, after having followed the laws of reflection.

Anyway, due to the short distances from the free surface, the first reflection path appear very similar to the direct path, if observed at distances from the source.

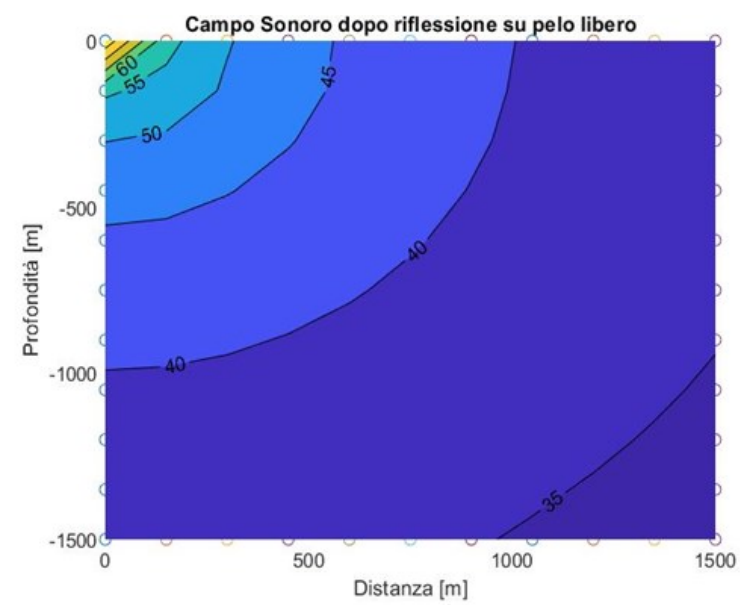

Fig. 14. Reflective field propagation

Each point of contact with the seabed is transformed into a virtual emission source, which falls within the propagation domain, after following the laws of reflection.

The contributions of the sound fields have been that summed from the energy point of view as reported in next picture (16), where a zoom limited at $500 \mathrm{~m}$ of depth and length is shown. 


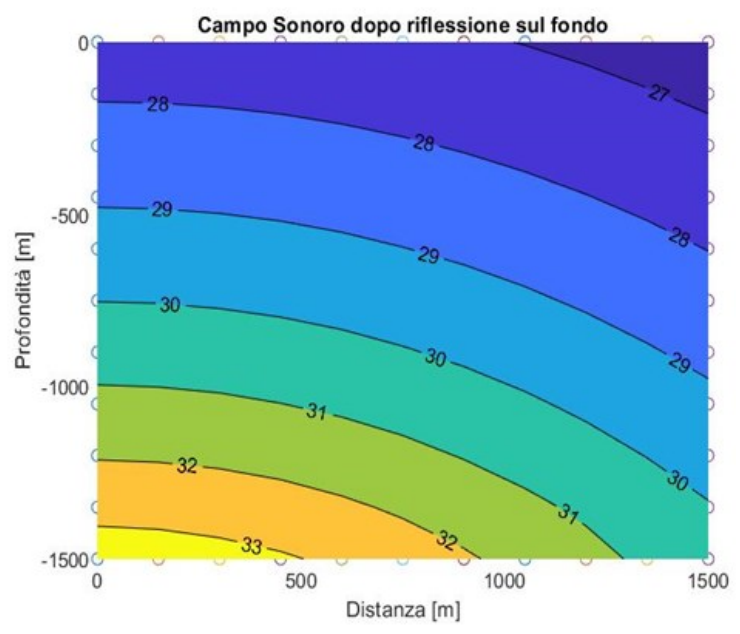

Fig. 15. Sea bed reflective field propagation

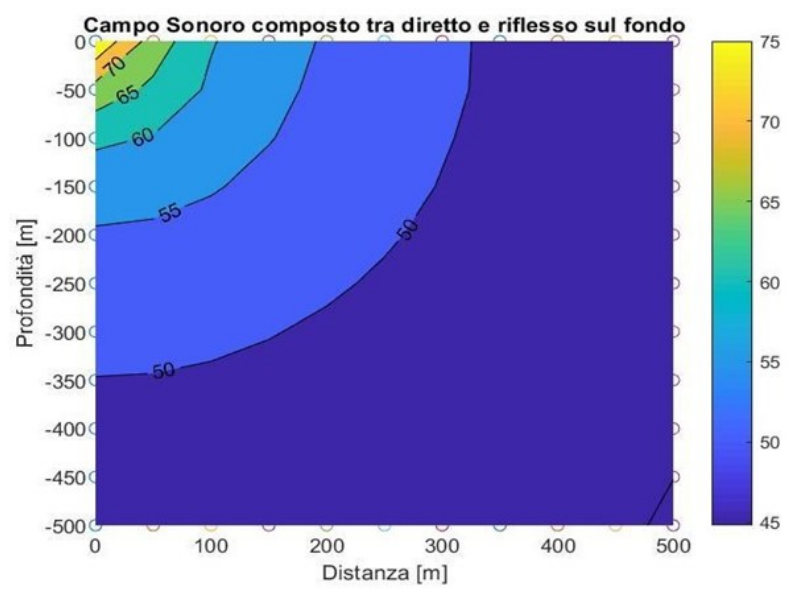

Fig. 16. Global SPL distribution

If the same picture is referred to a longer length it take evidence of the typical "cilinder like" attenuation path, generally reported for the underwater noise propagation.

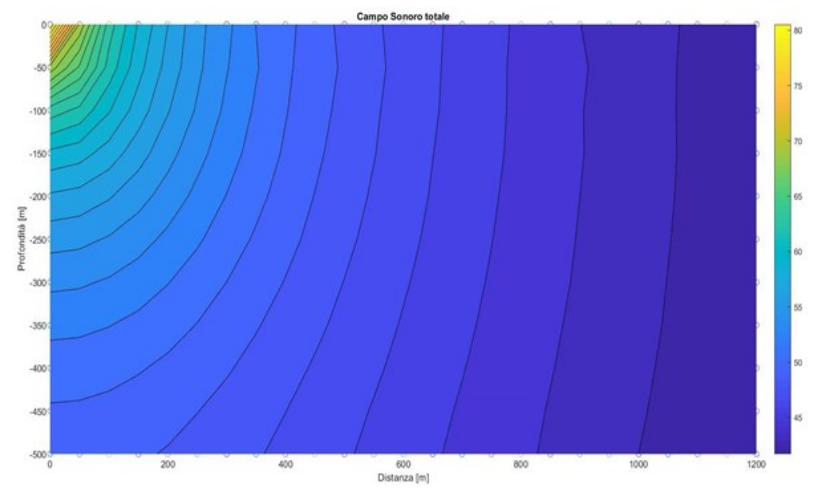

Fig. 17. Global SPL distribution

\section{Conclusion and future steps}

In the present work it has been studied the submarine noise pollution generated by the naval traffic.

To deal with this problem, one of the most widely used calculation tools for the study of sound propagation over long distances is the Ray Tracing method, a technique that exploits the optical acoustics analogy and is very exploited for the propagation of sound in the air. As explained along the paper, the propagation of sound in water is different from that in air because of the characteristics of the medium itself.

As part of the work, these considerations have been deepened from the theoretical point of view and a preliminary analysis was carried out of all the aspects that characterize the propagation in water, going to implement, in the Matlab environment, the characteristics of Ray Tracing and the environment of propagation going to characterize the propagation path. For the validation of the software we have imagined similar propagation scenarios to some found in the literature also validated experimental tests (Andersson, s.d.): these simulations have demonstrated a functionality of the code as expected.

In future developments, the code, as currently implemented, will be improved by implementing additional functions such as:

- Management of sound velocity profiles not linearly dependent on depth;

- Management of the seabed with presence of planoaltimetric irregularities;

- Management of further reflections in the evaluation of the sound field, for increasingly precise results;

- Management of multiple sources;

- Development in the third dimension.

\section{References}

1. Andersson, S., s.d. Modelling of Wave Propagation in Shallow Water Enviroment.

2. Audoly, et al., 2015. Mitigation Measures for Controlling the Ship Underwater Radiated Noise, in the Scope of AQUO Project.

3. Gaggero, Van Der Schaar, Rizzuto \& Andrè, 2012. Ship Underwater Noise Emissions: Uncertainties in the Measurements and in the Effects on the Marine Enviroment.

4. Jensen, F. B., 2011. Computational Ocean Acoustics.

5. Lurton, 2010. An Introduction to Underwater Acoustics: Principles and Applications.

6. Ross, 1987. Mechanics of Underwater Noise.

7. Tamà, Gaggero \& Rizzuto, 2015. Sound Propagation At Sea: A

8. Viscardi M., Coppola T., Quaranta F., Rizzuto E. 2017. On field experimental characterization of the ship sources of acoustic pollution within a commercial harbor, 24th Intern. Congress on Sound and Vibrations, July 2017, London GB.

9. Coppola T. Quaranta F. Rizzuto E., Viscardi M, D. Siano 2017. Development, calibration and validation of a model for the acoustic field generated by a ship at port, International Maritime Association of the Mediteranean, October 2017, Lisbon, Portugal. 